\title{
Políticas de empleo público en la provincia de Buenos Aires (1991-2016)
}

Public employment policies in Buenos Aires Province

Magdalena Gil García Unvestigaciones en Humanidades y Cie UNLP/CONICET
Fecha de recepción: 31.7.17

Fecha de aceptación 7.9.17

\section{Resumen}

A partir de la década de los noventa, en el marco de los procesos de reforma estatal y de modernización estatal, se implementaron políticas de empleo público que mostraron dos tipos de orientaciones diferentes: medidas que tenían como objeto el achicamiento del gasto en personal y la flexibilización laboral, y las orientadas a promover la profesionalización de los agentes públicos. Si bien la bibliografía especializada coincide en señalar que resultaron predominantes las estrategias de reducción de plantas de personal y flexibilización, la mayoría de los estudios se centra en forma exclusiva en los cambios operados en el ámbito nacional, existiendo menos antecedentes acerca de las modificaciones sucedidas en los niveles subnacionales. De esta manera, el presente artículo realiza un recorrido por las principales transformaciones en el empleo público de la Provincia de Buenos Aires desde 1991 dando cuenta del repertorio de políticas puestas en práctica y sus resultados, e ilustrando las tendencias prevalecientes en la materia. Así, el artículo abarcará cinco administraciones políticas: 1) el gobierno de Eduardo Duhalde (1991-1999);2) el de Carlos Ruckauf (1999-2001), 3) el de Felipe Solá (2002-2007); 4) el de Daniel Scioli (2007-2015); y 5) el actual, de María Eugenia Vidal (2015-actua- 
lidad). La hipótesis del trabajo es que el criterio "reduccionista" ha sido invariablemente utilizado durante todas las gestiones de gobierno, mientras que las iniciativas de profesionalización han tenido una escasa presencia en la agenda institucional y un carácter restringido, presentando resultado limitados.

Palabras clave: empleo público - provincia de Buenos Aires - reformas estatales - profesionalización - función pública

\section{Abstract}

From the nineties on, public employment policies were implemented as components of processes of state reform processes and modernization, aiming at both a reduction of budgets in labor employment together with labor flexibility and the promotion of professionalization of public servants. While specialized bibliography agrees in showing that downsizing and flexibility of employment conditions have been prevalent strategies, most of it focuses exclusively in changes conducted at the national level, with less attention addressed at transformations going on at sub-national levels. Thus, this article attempts to describe some of the principal transformations in public employment in Buenos Aires province since 1991, through an analysis of the different public employment policies implemented and their outcomes, in subsequen government administrations: 1) that of Eduardo Duhalde (1991-1999); 2) that of Carlos Ruckauf (1999-2001), 3) that of Felipe Solá (2002-2007); 4) those of Daniel Scioli (2007-11, 2011-2015); and current María Eugnia Vidal's (2015-present). Our hypothesis asseses that a "reductionist" approach has been invariably the leading one in all government administrarions, whilst professionalization initiatives have had limited incidence in public agendas, thus howing limited results.

Key words: public employment - Buenos Aires province - state reforms - professionalization - public function

\section{Introducción}

Como señala Orlansky (2006), la reforma del Estado incluye la reforma de las burocracias gubernamentales, de modo que las dinámicas y condiciones de trabajo del empleo público suelen ser analizadas tomando como punto de inflexión los procesos reformistas que se llevaron a cabo en la década de los noventa. Así, a partir de los procesos de reforma y modernización estatal, se implementaron políticas de empleo público que mostraron dos tipos de orientaciones: medidas que tenían como objeto el achicamiento del gasto en personal y la flexibilización laboral, y las orientadas a promover la profesionalización de los agentes públicos. Tales políticas comprendían normas, procesos, prácticas y actividades mediante las cuales el Estado regula y administra a su personal. De este modo, las estrategias "reduccionistas" se implementaron a través de medidas como los retiros voluntarios, jubilaciones anticipadas, congelamientos de cargos vacantes, leyes que pusieron en situación de disponibilidad a los agentes públicos, difusión de mecanismos de contratación por tiempo determinado, etc. Si bien la profesionalización resulta un concepto difuso, se la suele definir recurriendo a la Carta Iberoamericana de la Función Pública que funciona como un marco común en la región (Longo, 2008), y que entiende por profesionalización a la "garantía de posesión por los servidores públicos de una serie de atributos como el mérito, la capacidad, la vocación de servicio, la eficacia en el desempeño de su función, la responsabilidad, la honestidad y la adhesión a los principios y valores de la democracia" (CLAD-ONU, 2003: 3). La consolidación de un sistema de gestión del empleo público que promueva tales garantías puede realizarse a través de diferentes políticas: rediseño e implementación de los sistemas de carrera administrativa, introducción de mecanismos competitivos y transparentes de ascenso y promoción, profesionalización de sistemas de alta dirección pública, creación de cuerpos especiales basados en el mérito y la capacidad, planes de capacitación, gratificaciones al desempeño como parte de la política salarial, etc. La bibliografia coincide en señalar que las estrategias de reducción de las plantas de personal y de flexibilización laboral resultan predominantes, teniendo un fuerte impacto en el empleo público y dando como resultado que a menudo abandone el sector público el personal más capacitado (Oszlak, 2009). Sin embargo, la mayoría de los estudios se centran en forma exclusiva en los cambios operados en el ámbito nacional, existiendo menos antecedentes acerca de las transformaciones sucedidas en los niveles subnacionales.

El presente artículo realiza un recorrido por las principales transformaciones en el empleo público de la Provincia de Buenos Aires efectuadas desde 1991, dando cuenta del repertorio de políticas puestas en práctica y sus resultados. El trabajo se estructura de la siguiente manera: en la primera parte se presentará una caracterización del empleo público provincial, luego se analizarán las políticas implementadas en las cinco administraciones políticas que se desarrollaron entre 1991 y la actualidad, distinguiendo entre aquellas orientadas a la reducción del gasto en personal y la flexibilización laboral, y 
aquellas tendientes a la profesionalización de los agentes públicos. Finalmente en las conclusiones, se exponen las tendencias prevalecientes en el empleo público provincial, y se plantearan algunas de las consecuencias de dicha situación.

El trabajo requirió el análisis de fuentes secundarias (estadísticas oficiales, documentos de los organismos estatales, análisis de la normativa,) y la realización de entrevistas al personal técnico-profesional de los organismos rectores en materia de personal de la provincia y a representantes de los sindicatos estatales. En este punto debe señalarse que la ausencia de un sistema de información centralizado de personal en el ámbito de la provincia de Buenos Aires, impide la realización de un análisis exhaustivo y certero sobre la magnitud de los planteles del estado, sus características y distribución. No obstante, el presente trabajo toma los datos de diversas fuentes de organismos nacionales y provinciales para ilustrar algunas tendencias cuantitativas respecto de la planta ocupada.

\section{El empleo público en la provincia de Buenos Aires}

El empleo público provincial es un universo complejo y heterogéneo, puesto que abarca una amplia gama de actividades y estructuras. Al igual que en el resto de las provincias, el Poder Ejecutivo provincial es el que concentra la mayoría del empleo público; y en este la mayor cantidad de los cargos se encuentran comprendidos en cinco estatutos mayoritarios: el Estatuto Docente (55.3\%), el régimen de carácter general Ley 10.430 (24.6\%); la policía (11.7\%), el personal la Carrera Profesional Hospitalaria (2,7\%) y el Servicio Penitenciario (2.6\%) (Subsecretaria de Gestión Pública, 2005). Mientras que el $3 \%$ restante abarca al personal de diferentes regímenes: personal de actividades artísticas, técnicas y complementarias, personal de la Dirección deVialidad, personal de apoyo a la investigación, clero oficial, trabajadores de astilleros, personal de puertos, etc. Tal situación da lugar a una dispersión de las regulaciones laborales, de modo que el personal de la administración pública provincial está comprendido en el actual régimen básico de la Ley $N^{\circ} 10.430$, que es el estatuto general de aplicación para la mayoría de los agentes dependientes del Poder Ejecutivo y en 18 regímenes especiales agrupados de acuerdo con la especificidad de sus tareas (véase Tabla 1 en el Apéndice). La mayoría de estos regímenes tienen un carácter estatutario, lo que implica que resultan modificados por normas del Congreso o decretos el Poder Ejecutivo; mientras que sólo algunos sectores minoritarios cuentan con convenios colectivos de trabajo, ${ }^{1}$ los cuales permiten establecer la base común de derechos laborales a partir de la negociación entre empleadores y representantes de los trabajadores.

De esta manera, se entiende por "estatuto" al conjunto de normas legales que regulan la relación entre la administración pública y sus empleados, estableciendo los derechos, deberes y prohibiciones de los trabajadores del Estado. Como se ha señalado, el Estatuto de carácter general es la Ley 10.430, que rige para la administración pública central y para muchos órganos descentralizados, y fue sancionado en 1986 durante el gobierno de Alejandro Armendáriz (1983 - 1987), en reemplazo de la Ley 8721 sancionada por la dictadura militar, aunque con escasas modificaciones (Villarroel et al, 2012). Dicho marco legal se sustenta en ciertos principios: el ingreso en base a la idoneidad e igualdad de oportunidades, estabilidad basada en el buen comportamiento, derecho a la carrera a través de un sistema de calificaciones y concursos que ponderan el mérito y el desempeño satisfactorio. En este sentido, en relación a su diseño formal se trata de un modelo de carrera que tiende a organizar el empleo público a partir de un escalafón partiendo de la idea de que el funcionario se vincula de manera estable con la administración pública ocupando distintas categorías y funciones, y por lo tanto desarrollando su carrera profesional en dicho ámbito. Sin embargo, aunque la administración pública provincial posea una estructura formal acorde a este modelo, persiste una amplia brecha entre las reglas aparentes y las prácticas realmente vigentes. De este modo, el sistema de administración de personal provincial se caracteriza por un incumplimiento básico de la Ley en las disposiciones relativas a la carrera administrativa, de manera que los procesos de selección de personal resultan prácticas marginales y existe una falta de transparencia en las reubicaciones concretadas. La brecha existente entre las normativas que regulan las carreras de los agentes públicos y las prácticas imperantes no es un problema exclusivo de la provincia de Buenos Aires, sino que recorre diversas áreas y niveles del Estado. Si bien tal situación ha sido explicada por la bibliografia especializada atendiendo a diferentes variables ${ }^{2}$, la mayoría de los estudios coincide en señalar que los actores políticos carecen de incentivos para llevar a cabo iniciativas orientadas al establecimiento de sistemas de carrera basados en el mérito, ya que el incumplimiento de la normativa les permite preservar altos grados de libertad para manejar los ingresos, reubicaciones y promociones del personal. En este sentido, como señalan Dousset Farjat y Chudvnosvky (2016) las normas de promoción y ascenso en el empleo público constituyen "fachadas", es decir reglas de papel rutinariamente ignoradas, que los detentadores del poder tienen interés en mantenerlas por la legitimidad que otorgan pero ningún interés en hacerlas cumplir.

\section{El gobierno de Duhalde (1991-1995 / 1995-1999)}

Las reformas efectuadas en la órbita nacional en la década de 1990 tuvieron vastas consecuencias en las provincias, fundamentalmente porque implicaron una profundización de las políticas de descentralización iniciadas de los años setenta. ${ }^{3}$ Así, se transfirieron a las provincias la administración de las escuelas nacionales secundarias y para adultos y la supervisión de las escuelas privadas (Ley 24.049) y algunos hospitales públicos (Ley 24.061). ${ }^{4}$ Como consecuencia de este proceso las provincias debieron absorber el personal docente, técnico, administrativo y profesional de los servicios de salud y educación transferidos. ${ }^{5} \mathrm{Ha}$ sido señalado que el efecto de la descentralización no fue inmediato, puesto que la concentración de poder político del gobernador Duhalde le permitió contar con capacidad para demorar la transferencia efectiva. Asimismo, la aprobación 
del Fondo de Reparación del Conurbano Bonaerense con una asignación anual del gobierno federal de 6000 millones de dólares, facilitó el financiamiento de los servicios descentralizados (Aggof et al,2013). No obstante, la política de descentralización implicó que la provincia tuviera que administrar un aparato institucional más extendido, que derivó en una heterogeneidad de regímenes estatutarios y laborales y en un aumento significativo de los gastos en materia de personal. Así también, en dicho período fueron transferidos al ámbito de la provincia de Buenos Aires trabajadores de puertos, ferroviarios, astilleros y de casinos como parte de la reforma del Estado nacional. Como consecuencia de dicho proceso descentralizador la relación entre empleo público nacional y provincial cambió drásticamente, declinando marcadamente la incidencia ocupacional del sector público nacional.

De igual manera, y en consonancia con las transformaciones en el ámbito estatal nacional, la provincia de Buenos Aires llevó a cabo una serie de reformas aunque con dimensiones más acotadas y con menor presencia en la agenda institucional (Randazzo, 2004).Tal proceso reformista se inició a partir de la sanción de la Ley de Reconversión Administrativa (Ley 11.184) en 1991, que se orientaba a un reordenamiento de las estructuras burocráticas aplicando un criterio de racionalización tendiente a la disminución del gasto público (Erbetta, 2011). Dicha normativa autorizaba al Poder Ejecutivo a la intervención de diferentes entidades del sector público provincial a fin de racionalizar sus estructuras administrativas, optimizar recursos y servicios, y disponer la transferencia de las actividades pertinentes al sector privado. Con estos fines se creó el Consejo Asesor de Reforma del Estado y Procedimiento Administrativo, CAREPA (Decreto N¹8/91) el cual tenía como objetivo atender todos los temas relacionados con la reforma del Estado, los procedimientos administrativos y los regímenes estatutarios que hacen a la administración de gobierno y a la racionalización administrativa.

En materia de empleo público la Ley de Reconversión Administrativa puso en situación de disponibilidad a los trabajadores estatales de la administración central, descentralizada y de las empresas públicas y entidades financieras de la provincia. Esto significaba que el Poder Ejecutivo estaba autorizado a reubicar a los agentes públicos en cargos de igual o superior jerarquía, reasignarles destino o declararlos "prescindibles". Dicha normativa tuvo una orientación reduccionista, ya que establecía mecanismos para disminuir la dotación de personal a través de jubilaciones anticipadas, retiros voluntarios y la prescindibilidad $^{6}$ de los agentes públicos que no fuesen confirmados o reubicados al finalizar la disponibilidad. En este sentido, la prescindibilidad constituía una "norma excepcional de despido" (Orlansky, 2006), dado que si en el marco de la restructuración administrativa y/o cierre de organismos el empleado no conseguía la reubicación, quedaba sin empleo. Tal situación se complementó con el congelamiento de los cargos vacantes en planta permanente y en planta temporaria, exceptuando a los contratos (Decreto 1559/92). Así, si bien en el Estatuto - Ley se contemplaba la posibilidad de establecer contrataciones de personal por tiempo determinado para la realización de tareas de carácter eventual o temporario, es en este contexto que dichas figuras contractuales se convierten en vías de ingreso privilegiadas a la administración pública encubriendo puestos de carácter permanente.

La estrategia provincial no era exclusivamente el reordenamiento administrativo de las estructuras burocráticas sino también configurar un nuevo rol del Estado, desplazando actividades hacia el ámbito privado. De este modo, durante la gestión Duhalde se llevaron a cabo las privatizaciones de la Empresa Social de Energía de Buenos Aires (ESEBA) en 1997 y de Obras Sanitarias de Buenos Aires (OSBA) en 1999.

Dichas políticas "reduccionistas" se combinaron con un conjunto de iniciativas orientadas a la profesionalización de los agentes de la administración pública. En 1991 se creó el Instituto Provincial de Administración Pública (IPAP) (Decreto 2688/92) encargado de la capacitación en el ámbito provincial, en reemplazo de la Escuela Superior de Administración Pública -dando cuenta de una jerarquización institucional de la problemática-. Asimismo se sancionó la Ley de Evaluación de Desempeño (Decreto 4608/93). Dicha normativa tenía como finalidad motivar el rendimiento de los agentes públicos mediante procedimientos que determinaban la eficiencia individual de los agentes públicos, considerando la calidad del trabajo desempeñado, el conocimiento de la tarea, la cantidad de trabajo, la iniciativa, responsabilidad, receptividad, versatilidad, asistencia y puntualidad del agente, sus relaciones personales. A partir de las calificaciones obtenidas en dicha evaluación, se establecía un sistema anual de incentivos económicos consistentes en un número decreciente de sueldos básicos. La propuesta de introducir mecanismos de pago variable que premien el rendimiento individual en el puesto de trabajo derivaba en gran medida de las ideas del New Public Management ${ }^{7}$, cuerpo doctrinario imperante en las reformas administrativas de numerosos países de la Organización para la Cooperación y el Desarrollo (OCDE) ${ }^{8}$ (Iacovello y Strazza, 2011). Sin embargo, desde su sanción normativa la evaluación del desempeño fue escasamente utilizada. En este sentido algunos autores concluyen que las reformas vinculadas a la evaluación del desempeño son un claro ejemplo de la distancia que media entre el discurso formal y su aplicación (Longo y Ramio, 2008). De acuerdo a Oszlak (2009), el uso de estos sistemas suele responder a un proceso de mimetismo institucional, pero su implementación encuentra numerosos obstáculos debido a las dificultades en su administración, los altos porcentajes de apelación que poseen sus resultados y los conflictos internos que generan entre el personal y los evaluadores.

Debe recordarse asimismo que la Ley de empleo público establecía procesos de calificación de personal que funcionaban como el fundamento de las decisiones de promoción. Sin embargo, durante todo el período persistió el problema de incumplimiento de la ley en las disposiciones relativas a carrera debido a la ausencia de calificación del personal, ${ }^{9}$ 
la cancelación de los concursos y las irregularidades en los mecanismos de promoción, agravadas en el marco de las reubicaciones indiscriminadas habilitadas por la Ley de Reconversión Administrativa. De esta manera, aunque la creación del IPAP puede haber significado un impulso a la capacitación, la participación en dichas instancias quedó librada al interés individual de los agentes públicos, dado que la cancelación de la carrera administrativa tiende a generar escasos incentivos a la formación del personal.

Aunque prevalece la falta de información sistematizada sobre la magnitud de los planteles de los estados provinciales, es posible realizar algunas estimaciones respecto de la dinámica con base en los datos de la Dirección Nacional de Coordinación Fiscal con las Provincias (DNCFP). Así, en materia de transformaciones cuantitativas, hubo un crecimiento significativo de la planta ocupada durante las dos gestiones del gobernador Duhalde, produciéndose la mayor variación interanual en el año 1995 (11,5\%), debido posiblemente a la dilación del proceso descentralizador. De igual manera, en contraste con la retórica de achicamiento del Estado característica de la época durante todo el período se observa un aumento de las dotaciones de personal, que registran un crecimiento del 43\% en 1999 respecto al 1991 (Tabla 2 y Gráficos 1 y 2 en el Apéndice)).

\section{Los gobiernos de Carlos Ruckauf (1999-2002) y Felipe Solá (2002-2007)}

En julio 2001, el marco de la crisis social y económica, se dictó la emergencia administrativa, económica y financiera de la provincia mediante la Ley 12.727. Junto con normas complementarias (Decreto 2040/01), la ley autorizó al Poder Ejecutivo a disponer la rescisión de contratos de obra y de servicio suscriptos por la Administración Pública provincial y a renegociar sus condiciones. Con la intención de reducir el gasto público se sancionó el congelamiento de vacantes a partir de la suspensión de las designaciones de personal en planta permanente y temporaria de toda la Administración Pública (Decreto 2658/00). De esta manera el empleo público provincial en el año 2002 descendió un 5,25\% respecto al año 2000, de acuerdo a datos de la DNCFP (Tabla 2 y Gráfico 2 en el Apéndice). Las iniciativas de ajuste se manifestaron además a través de una política salarial que implicó la reducción de retribuciones brutas totales, mensuales, habituales y regulares y permanentes, y el sueldo anual complementario del total del personal de los organismos provinciales y de los beneficiarios del sistema previsional ${ }^{10}$ (Art. 15 Ley 12.727). Al mismo tiempo, dicha Ley dispuso el pago parcial de haberes y retribuciones con letras de la tesorería para la cancelación de obligaciones (patacones) y con Letras de Cancelación de Obligaciones Provinciales emitidas por el gobierno nacional (LECOP) (Randazzo, 2004)

Cuando a comienzos de enero 2002 Eduardo Duhalde asumió la presidencia interina, el gobernador Carlos Ruckauf renunció a la gobernación para asumir el Ministerio de Relaciones Exteriores del nuevo gobierno. Fue sucedido por el vicegobernador Felipe
Solá, en un contexto de profunda crisis financiera. A esta situación se sumaba una gran conflictividad gremial dado que las medidas de emergencia produjeron múltiples cuestionamientos de los sindicatos estatales. Con el mismo espíritu reduccionista que su predecesor, Solá creó el sistema de Unidades Retributivas por Productividad y Eficiencia (URPE), que consistía en un estímulo económico destinado a premiar la productividad y la eficiencia en el desempeño de tareas especiales, y que se proponía reemplazar las prácticas informales de compensación salarial sobre la base de viáticos y horas extras. Dicha iniciativa se orientaba al ordenamiento de los sistemas salariales, disminuyendo la distribución arbitraria de tales asignaciones, pero en la práctica el uso de las URPES se desvirtúo y terminó superponiéndose a los otros mecanismos de compensación.

Una vez que la provincia salió de la emergencia económica, se presentó un contexto más favorable para llevar a cabo reformas en materia de empleo público con otra orientaciones. Una de las transformaciones más importantes en materia de empleo público durante el gobierno de Solá fue la apertura de las negociaciones colectivas en 2004 y su reglamentación en 2006 (Ley 13.453). ${ }^{11}$ De esta manera, si bien el derecho a la negociación colectiva de los trabajadores estatales había sido reconocido en la Constitución Provincial de 1994, pudo ser ejercido plenamente recién diez años después. ${ }^{12} \mathrm{La}$ institucionalización de las relaciones laborales fue un hecho significativo porque abrió la discusión paritaria para la recuperación salarial y permitió la construcción de un espacio de consenso entre el gobierno y los representantes gremiales, para el diseño de políticas de empleo público encaminadas a la reactivación de la carrera administrativa. De esta manera, en dicho ámbito se creó una comisión de Carrera Administrativa con la finalidad de avanzar en el rediseño del sistema de carrera. La comisión estaba integrada por funcionarios políticos y personal técnico-profesional del Ministerio de Trabajo y la Subsecretaria de Gestión Pública, y por los representantes gremiales de la Asociación de Trabajadores del Estado (ATE) y la rama provincial de la Unión del Personal Civil de la Nación (UPCN). En ese marco se elaboró y discutió un proyecto que establecía un nuevo modelo de carrera profesional administrativa basado en la valoración de competencias como requisitos de ingreso, ascenso y promoción (Villarroel et al, 2012). El sistema de carrera propuesto tendía a poner de relieve la valoración de los "conocimientos en acción" tanto provinieran de la educación formal o de la experiencia adquirida en la administración pública y otros espacios laborales; y se basaba en la descripción de puestos y competencias. Así, se tomó a las competencias como el criterio fundamental para determinar las capacidades requeridas de los individuos en función del puesto de trabajo, definiéndoselas como "conjunto de saberes que las personas expresan en situaciones laborales y sociales, lo que involucra valores, conocimientos y actitudes, y hacen a un correcto desempeño en un puesto de trabajo" (Quintans y García, 2013: 7). Asimismo, se distinguieron tres tipos de competencias para llevar cabo los procesos de descripción de los puestos: las ético-institucionales, referidas a los valores y concepciones de la persona respecto la sociedad y las instituciones; las técnico-profesionales, relativas a los conoci- 
mientos específicos para el cumplimiento de las acciones correspondientes a un puesto de trabajo determinado; y las actitudinales, relacionadas a las actitudes y estrategias que las personas ponen en juego en la relación con el entorno laboral (Subsecretaria de Gestión Pública, 2006)

Como resultado de estas iniciativas se reactivaron progresivamente, a partir del año 2005 , los procesos de selección para cargos jerárquicos interinos (subdirectores y jefes de departamento), con base en pautas establecidas en una guía orientadora elaborada por la Subsecretaria de Gestión Pública (Resolución Nº 08/07) y consensuada con los representantes sindicales. El desplazamiento semántico hacia la noción de proceso de selección, se debió a la necesidad de diferenciar dicho mecanismo del procedimiento de los concursos sancionados en la Ley, que requerían la realización de los procesos de calificación del personal, los que siguieron suspendidos durante toda la etapa analizada.

Otra iniciativa orientada a la profesionalización fue la implementación del programa de Agentes de Modernización (Decreto 540/03) que tenía como objetivo la formación de un cuerpo profesional "altamente capacitado en el conocimiento de normas y sistemas que rigen el funcionamiento de los organismos provinciales y para la modernización de la administración pública provincial con nuevas técnicas de gestión" (Subsecretaría de Gestión Pública, 2005). El programa estaba dirigido a los empleados de planta permanente de la administración provincial que, luego de un proceso de selección de acuerdo a determinados requisitos, ${ }^{13}$ atravesaron un intenso proceso de formación y evaluación coordinado por el IPAP. Dicha experiencia apuntaba a la creación de una burocracia profesionalizada para la realización de tareas de asesoramiento técnico-profesional en diferentes organismos del Estado, inspirándose en la experiencia de los Administradores Gubernamentales de la administración pública nacional. Sin embargo, a diferencia de estos, el cuerpo de los Agentes de Modernización tenía una escala más reducida y carecía de un escalafón propio. Dicha experiencia tuvo dos ediciones: la primera en el año 2003 durante la gestión de Florencio Randazzo en la Secretaria para la Modernización del Estado; la segunda se realizó en 2005, impulsada desde la Subsecretaria de Gestión Pública por la Ingeniera Claudia Bernazza. Como resultado de ambas experiencias resultaron designados veintiún profesionales que pasaron a constituir el cuerpo de los Agentes de Modernización. Se ha señalado sin embargo que el programa no tuvo el éxito esperado debido a la falta de recursos y de apoyo puestos a su disposición, de modo que terminó siendo abandonado el año 2009 y reemplazado por otro de similares características (Piana, 2012).

Pese a los avances mencionados, las iniciativas de reactivación de la carrera administrativa dieron resultados limitados ya que no logró implementarse el nuevo sistema de carrera, y si bien se restablecieron algunos procesos de selección para el agrupamiento jerárquico su impacto fue acotado a ciertos organismos. De esta manera persistió el pro- blema de incumplimiento básico de la ley en lo relativo a los ascensos y promociones para el resto de los cargos que componen el escalafón. Ante la imposibilidad de dar una respuesta global a la problemática de la carrera administrativa, y como compensación al estancamiento escalafonario de los agentes públicos, en 2007 se realizó una reubicación que tenía como criterio central la antigüedad (Decreto 3283/07). Asimismo, durante todo el gobierno de Solá permaneció la apelación a la lógica reduccionista a partir de la prórroga del congelamiento de vacantes mediante sucesivos decretos anuales. Ello no generó una disminución de las plantas de personal, las que, al contrario, mostraban a fines del 2007 un crecimiento de casi 28,5\% respecto de 2002 (ver Tabla 2 y Gráfico 1). También tuvo como consecuencia el mayor crecimiento de modalidades de contratación que carecen de estabilidad, frente a las de carácter permanente.

\section{El gobierno de Daniel Scioli (2007-2015)}

En este doble periodo de gobierno nos encontramos con algunos esfuerzos fragmentarios en diferentes direcciones. En materia de profesionalización, en noviembre 2008 la Secretaría de Modernización del Estado avanzó en la elaboración de un Anteproyecto de Ley Marco de Empleo Público para reemplazar a la ley 10.430, que fue sometido a consideración de los representantes sindicales en el ámbito de la negociación colectiva La Comisión de Carrera Administrativa pasó a denominarse "Comisión Redactora", encargada de elaborar y discutir una nueva Ley Marco. En dicho ámbito en se consensuaron 74 artículos de la nueva Ley de Empleo Público: una nueva carrera administrativa basada en el mérito y en la formación permanente; un nuevo escalafón eliminando las categorías más bajas; derechos y obligaciones de los empleados públicos (Villarroel et al, 2012). Con relación a la carrera administrativa, se seguía trabajando en un esquema similar al período anterior, que establecía un desarrollo horizontal y vertical basado en la valoración de competencias, la evaluación de desempeño y la realización de concursos (Mouriño, 2009). La Comisión Redactora dejó de funcionar a fines de 2009 debido al abandono por parte de las autoridades políticas provinciales, quedando pendiente la sanción de la Ley Marco y desapareciendo de la agenda de gobierno las iniciativas de recuperación de la carrera administrativa.

El programa de Expertos en Gestión Pública reemplazó al cuerpo de Agentes de Modernización. Igual que su predecesor estuvo dirigido a los profesionales de la planta permanente de la administración pública provincial, los cuales fueron preseleccionado sobre la base de ciertos requisitos, y atravesaron un ciclo de capacitación en el período 2010-2011. A diferencia de la experiencia anterior, el diseño y dictado del programa de especialización no estuvo a cargo del IPAP, sino de la Universidad Nacional Tres de Febrero, de modo que los agentes recibieron una formación de posgrado acreditada por dicha universidad ${ }^{14}$. Como resultado del proceso formativo fueron seleccionados veinticinco agentes sobre la base de un orden de mérito, otorgándoseles un cargo de 
Expertos en Gestión Pública de la planta permanente sin estabilidad, con rango y remuneración equivalente al de Director, con una duración de cinco años. El programa tropezó con varios obstáculos debido a la alta rotación de las autoridades encargada de dirigirlo, de modo que recién en el año 2013 se concretaron las designaciones de los profesionales que integran el cuerpo de expertos (Decreto 209/13). Dichos agentes tienen como función principal brindar apoyo técnico a diferentes organismos provinciales y municipales para la implementación de mejoras en la gestión. No obstante se trata de una escala reducida en relación a las dotaciones de la administración pública provincial, y el cuerpo de profesionales no cuenta con un escalafón específico, siendo designados para dichas funciones por un período determinado. Esto tiende a otorgar inestabilidad al programa y dificulta la consolidación de una burocracia profesionalizada con relativo aislamiento de las interferencias y fluctuaciones de la política.

Con la sanción del Decreto 3/12 se restablecieron mecanismos de reducción de los planteles de recursos humanos. Dicha norma estableció que los cargos vacantes serían absorbidos por el Ministerio de Economía y que toda nueva designación o recategorización requeriría la aprobación de dicha Jurisdicción, lo que generaba nuevos obstáculos para la concreción de tales movimientos. De este modo, con dicho decreto se pretendía preservar el equilibrio presupuestario, limitando tanto las incorporaciones de personal a la planta permanente y temporaria como los ascensos en la carrera administrativa. Así, si bien hubo durante los dos periodos de Scioli un crecimiento significativo del empleo público, en el año 2014 se observa una caída del 1,26\% respecto del año 2012 (DNCFP) (Tabla 2 y Gráfico 2).

\section{El gobierno de Vidal (2015-actualidad)}

En el año 2015, frente al cambio de gobierno de distintos signo político-partidario en diferentes niveles del Estado (nación, provincia y municipios), la problemática de empleo público cobró relevancia en la agenda del estatal, generándose debates públicos respecto a su magnitud, politización y el incumplimiento por parte de los trabajadores de sus obligaciones laborales.

En el ámbito provincial, en el marco de la declaración de la Emergencia Administrativa y Tecnológica (Ley 14.815), ${ }^{15}$ el gobierno priorizó la implementación de medidas orientadas a la flexibilización y a la reducción de los planteles del Estado, al disponer el congelamiento de vacantes y suspender las designaciones en planta permanente y transitoria de todas las jurisdicciones de la administración pública con el objeto de "superar la insuficiencia de recursos económicos y financieros" (Ley 14.818). La ley creó un nuevo régimen de contratación de servicios, que establece que la relación entre el contratado y la administración no se regirá por la Ley de empleo público sino por las cláusulas del contrato, y que la celebración del mismo no generará una expectativa o derecho a prórroga, ni creará una relación laboral de dependencia. Como hemos señalado, desde la década de los noventa constituye una práctica habitual en el ámbito provincial la incorporación de personal para la realización de tareas de carácter permanente a través de contratos de locación de servicios. Sin embargo esta nueva figura contractual creada en el marco de la emergencia, a diferencia de los contratos de servicios establecidos en el Estatuto General Ley 10.430, carece de aportes previsionales y coberturas médicas a cargo del empleador, así como de los derechos colectivos de representación y cobertura sindical. Así, se trata de un proceso donde se transfieren responsabilidades y riesgos desde el Estado empleador hacia el trabajador contratado bajo "la figura de autónomo" en el marco de un proceso de individuación de las condiciones de trabajo y relaciones laborales (Poblete, 2013). Según entrevistas realizadas en el año 2017 a representantes gremiales y a personal técnico-profesional de las áreas de recursos humanos, la utilización de la normativa ha permitido que se constituyan dos estratos diferenciados de trabajadores contratados: una cúpula flexibilizada con altas remuneraciones y permeable a los requerimientos del personal político, que tiende a conformar una burocracia paralela, y un sector de trabajadores con funciones y salarios similares al personal de planta permanente y con formas de contratación altamente precarizadas.

Asimismo entre las medidas orientadas al recorte de los gastos en materia de personal se sancionó el Decreto 272/17 de similares características al Decreto 3/12 de la gestión Scioli. El nuevo instrumento establece que los cargos vacantes serán absorbidos por el Ministerio de Economía y puestos a disposición del Poder Ejecutivo, obstaculizando su utilización por parte de las diferentes jurisdicciones para concretar incorporaciones de personal y recategorizaciones

\section{Conclusiones}

En las páginas precedentes, hemos realizado un recorrido por las principales transformaciones en el empleo público provincial desde 1991 hasta la actualidad, dando cuenta del repertorio de acciones y programas puestas en práctica por las sucesivas administraciones de la provincia de Buenos Aires. Desde la década de los noventa las políticas implementadas en materia de empleo público han mostrado dos orientaciones diferentes: políticas orientadas al achicamiento del gasto y la flexibilización laboral; y medidas orientadas a promover la profesionalización de los agentes públicos. La provincia muestra una exigua innovación en la Ley de empleo público y en las normativas que regulan la carrera administrativa, en contraste con el ámbito de la administración pública nacional, donde los "shocks de reforma" se expresaron en diversas actualizaciones del sistema de carrera para el personal de la administración central ${ }^{16}$. Asimismo, en el ámbito provincial las estrategias reduccionistas fueron invariablemente utilizadas durante todo el período analizado a través de diversos mecanismos: la situación de disponibilidad de los agentes públicos, los sucesivos congelamientos de vacantes, las restricciones en 
materia de recursos humanos, y la creación de nuevas figuras contractuales por tiempo determinado. Mientras que las iniciativas de profesionalización han tenido en la agenda institucional una presencia limitada a ciertos períodos de gobierno, un carácter restringido y con escasos resultados.

Fue en el gobierno de Solá (2002-2007), cuando se implementaron de modo sistemático varias iniciativas orientadas a promover la profesionalización de los agentes públicos: rediseño del sistema de carrera administrativa, realización de procesos de selección para cargos jerárquicos interinos, creación de un cuerpo profesionalizado para la realización de tareas de asesoramiento técnico-profesional en el marco de la modernización estatal. No obstante tuvieron alcance limitado, ya que el nuevo sistema de carrera nunca logró aprobarse formalmente y con el cambio de gestión gubernamental los procesos de selección fueron perdiendo el impulso inicial y se constituyeron en prácticas marginale de algunos organismos. Frente a tal situación, la experiencia orientada a la profesionalización del personal que logró implementarse, y aún continúa vigente, es el programa de Expertos en Gestión Pública durante el gobierno de Daniel Scioli. Se trata de programa de formación y jerarquización de la planta permanente del Estado, en oposición a la tendencia a contratar personal externo especializado, pero tiene una escala reducida con relación a los planteles estatales y no logra dar una solución general a la problemática de la cancelación de la carrera administrativa.

Frente a la escasa presencia en la agenda de iniciativas orientadas a la profesionalización de los agentes públicos, se observa durante el período analizado la preservación de un statu quo en las políticas de empleo público caracterizado por el incumplimiento de las normativas que regulan la carrera administrativa y la apelación a las estrategias "reduccionistas".

Así, al igual que en otros ámbitos en la provincia de Buenos Aires "la reducción de costos pasa a ser el criterio 'estratégico' por excelencia” (Iacovello et al, 2003), constituyéndose en la línea de acción invariablemente implementada en materia de gestión de personal, lo cual tiene una serie de efectos en la evolución de las dotaciones de personal, las formas contractuales imperantes, la carrera de los empleados públicos y sus salarios. Para comprender la apelación frecuente a este criterio, es necesario recordar la estrecha relación que existe en el sector público entre condiciones de trabajo y las cuentas públicas, puesta de relieve por la bibliografía especializada (Diana Menéndez, 2009; Barzolla et al, 2012). Así, la provincia de Buenos Aires se encuentra tendencialmente afectada por "la naturaleza rígida de los gastos públicos provinciales (servicios públicos personales) y la vulnerabilidad de los ingresos de la provincia, una de cuyas fuentes es la magra recepción relativa de recursos coparticipables, y otra, la caída relativa de algunos tributos provinciales" (Aggof et al, 2013:

30). De esta manera, en épocas recesivas el presupuesto puede funcionar como un juego de suma cero, lo que es utilizado como un argumento desde el gobierno para llevar a cabo estrategias orientadas a la austeridad del gasto y a la flexibilización laboral.
Pese a la implementación de diferentes medidas orientadas acotar el gasto y preservar el equilibrio presupuestario, no se logró la disminución de las plantas de personal debido a que la retracción se observa sólo en los períodos más agudos de las crisis, pero superado éstos se compensa con un nuevo crecimiento de las dotaciones (Gráficos 1 y 2). Así, entre 1991 y 2015 las plantas de personal aumentaron 139\%. (DNCFP). No obstante, como resultado de los sucesivos congelamientos de vacantes en planta permanente, mientras en el año 1991 sólo 12\% de los cargos presupuestados era de carácter temporario, en la actualidad representan $21 \% .{ }^{17}$ Aunque no contamos con datos precisos, esa cifra aumenta si incluimos a los trabajadores contratados bajo diferentes modalidades tales como el régimen del monotributo, contratos de sala y escenario del Instituto Cultural, y becas del Ministerio de Salud. ${ }^{18}$. Algunas de estas modalidades de contratación por tiempo determinado implican un proceso de precariedad laboral, puesto que carecen de múltiples derechos laborales tales como: régimen de licencias, aportes previsionales y coberturas médicas a cargo del empleador, representación y cobertura sindical.

Asimismo, en tanto realizar una carrera administrativa implica haber logrado reconocimientos morales y materiales fundados en el mérito y la equidad, el mantenimiento de dicho statu quo tiende a obstaculizar que la administración pública provincial constituya un ámbito de trabajo que permita realizar trayectorias de crecimiento profesional y laboral. 
${ }^{1}$ Personal Transferido de la Administración General de Puertos Sociedad del Estado a la Dirección Provincial de Actividades portuarias; Personal Ferroviario Transferido; Personal de Astilleros Río Santiago; Personal de Organismo de Control de la Energía Eléctrica de la Provincia de Buenos Aires.

Algunos estudios han puesto de relieve que diferentes factores, con distinto peso segun el ámbito y el caso funcionarian como obstaculizadores de la implementación efectiva de los sistemas de carrera basados en et mérito entre los que se destacan: el diseño institucional del sistema politico, las características de la cultur organizacional, la ausencia de coaliciones promotoras, los intereses opuestos, la falta de voluntad política, lo actores tecrnicos, etc. (Llano Carbonel, 207, Longo y Ramio, 20 s, Geddes, 1996, Oszlak, 2009). (a) potale, de electricidad etc. anteriormente administo dos por el sobierno nacionl" (2006: 52). de ag

Orlansky (2006) señala que la descentralización de los servicios de educación implicón de la Ley 21.883 26.117 cargos docentes y no docentes al ámbito de la provincia de Buenos Aires.

En julio de 1993 se deja sin efecto la prescindibilidad de los agentes públicos (Decreto 2489/93), pero se mantiene la aplicabilidad de las reasignaciones de destino, rotaciones y/o reubicaciones del personal, régimen jubilatorio de excepcion, pasividad anticipada y retiros voluntarios (Decreto 952/94), que se extendió durante el periodo de Reconversión Administrativa (Ley 11.489) hasta timales de 1994.

blideario del New Public Management - que está lejos de ser un modelo coherente- inspiró a partir de la década de los ochenta diferentes programas de modernización y reforma de las administraciones públicas.

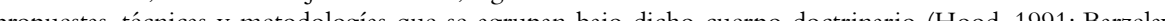
2001: Pollit y Bouckert 2000; Guerrero 2003: López 2005). encuentra incluido dentro de las líneas de acción orientadas a la "flexibilización". Según dicho autor la noción de flexibilización ha sido el lema acuñado por la OCDE para sintetizar las orientaciones 政

"El abandono de los mecanismos de calficicación de personal no parece responder a un hito normativo o instituciona preciso. En las entrevistas realizadas algunos funcionarios señalaron que desde la vuelta a la democracia estas prácticas no se realizaron, mientras que otros sostuvieron que hubo algunas calificaciones a fines de la decada de los ochenta. "Esta politica tuvo su correlato en el ámbito nacional puesto que, en el marco de la recesión económica de lo años 2000 y 2001, se establecieron recortes de los salarios publicos nominales entre el $12 \%$ y el $15 \%$ durante $l_{\text {a }}$ ${ }_{11}^{1}$ Bruno (2008) y Vilharoel et al (2012) ba a alizado lo carcterísticas que asume el sistema de negociación colectiva provincial dando cuenta de los contenidos sometidos a la agenda los ámbitos, actores, dinámicas y principales resultados.

negociaciones colectivas en el abámbito existido iniciativas tendientes a la regulación e institucionalización de las creto $\mathrm{N}^{\circ} 1198$ que reconocía al Poder Ejecutivo la potestad de recurrir a la negociación colectiva en numerosa materias, pero que nunca logró aplicarse. Así también, en el ano 2000 la Legislatura Provincial sancionó una ley sobre negociación colectiva que finalmente resultó vetada por el gobernador Carlos Ruckauf (Bruno, 2008). ${ }_{13}^{13}$ Para postularse se les exigía: tener más de treinta años de edad, cinco de antigüedad en la administración pública, titulo universitario o de estudios superiores no inferiores a cuatro años, preferentemente con formación de postrado y especialzación á ác perincis.

Gestión Pública de la Universidad Nacional de Tres de Febrero.

A su vencimiento dicha Ley fue prorrogada mediante el Decreto 53/17.

La primera modificación del sistema de carrera en el ámbito de la administración pública nacional fue en 1991 a través de la implementación de un nuevo escalafón denominado Sistema Nacional de Profesión Administrativa (SINAPA), modificado por el Sistema Nacional de Empleo Público (SINEP) en 2008. La bibliografa señala que aunque muestran debilidades en su implementación, generaron avances en el establecimiento de mecanismos de evaluación de desempeño e incentivos a la profesionalización y capacitación (Iacovello, $e$ al, 2009; Chudvnosvky y Dousset Farjat 2016

No existen regitros estadíticos sobre dichas mod ificables en las parat

\section{Referencias}

Aggof, Sergio; Bretanau, Julián; Foglia, Carolina (2013) El gobierno de la educación pública bonaerense entre 1983 y 2010: momentos, actores $\gamma$ tensiones. Ponencia presentada en el XI Congreso Nacional de Ciencia Política, Universidad Nacional de Entre Ríos. Inédita.

Barzelay, Michael (2001) The new public management: Improving Research and Policy Dialogue. Estados Unidos: University of California Press.

Bruno, Mirta (2008) Negociación colectiva en el sector público. Documento de la Subsecretaria de Modernización del Estado Provincia de Buenos Aires (en línea). Disponible en www.claudiabernazza.com.ar/ssgp/html/biblioteca/negociacion_colectiva.dod

Chudnovsky, Mariana y Doussett Farjat, Micaela (2016) "Cuando la falla en la implementación de las reglas se busca modificar con más reglas. La historia de las reformas del empleo público en Argentina”. Desarrollo Económico 55 (217):315-341.

CLAD-ONU (2003) "Carta Iberoamericana de la Función Pública”. Santa Cruz de la Sierra, Bolivia. Documento disponible en www.clad.org.ve

Diana Menéndez, Nicolás (2009). Condiciones de trabajo y precarización laboral en el Estado. El caso de los trabajadores del Ministerio de Trabajo, Empleo y Seguridad Social de la Nación (tesis de Doctorado). Facultad de Ciencias Sociales, Universidad de Buenos Aires.

Erbetta, María Cecilia (2011) "La reconversión del estado durante la primera gobernación de Duhalde en la Provincia de Buenos Aires". Revista trabajo y sociedad 15 (16): 287-298.

Geddes, Barbara (1996) Politician's Dilemma: Building State Capacity in Latin America. Berkeley: University of California Press.

Guerrero, Omar (2003) "Nueva Gerencia Pública. ¿Gobierno sin Política?”. Revista Venezolana de Gerencia 8 (23):379-395.

Hood, Christopher (1991) “A public managament for all seasons?”. Public Administration, Vol. 69: 3-19.

Iacovello, Mercedes; Zuvanic, Laura y Tomassi, Mariano (2009) "El servicio civil en a Argentina: disfuncionalidades y núcleos centrales de su abordaje". En Guillermo Schweinhem, ed., Estado y administración pública: críticas, enfoques y prácticas en la Argentina actual. Buenos Aires. Asociación Argentina de Estudios en Administración Pública, págs. 175-200. 
Iacoviello, Mercedes y Strazza, Luciano (2011) "De partidocracias rígidas a meritocracias flexibles en América Latina". Documentos y Aportes en Administración Pública y Gestión Estatal (DAAPGE), año 11 (16): 51-95.

Llano Carbonel, María Mercedes (2016). Burocracia pública y sistema político en América Latina: factores asociados a la politización de los sistemas de gestión de empleo público en la región (tesis de doctorado). Universidad Complutense de Madrid.

Longo, Francisco (2004) Mérito y Flexibilidad. La gestión de las personas en las organizaciones del sector público. España: Paidos.

Longo, Francisco (2008) "La profesionalización del empleo público en América Latina. Estado de la cuestión”. En F. Longo y C. Ramio, La profesionalización del empleo público en América Latina. Barcelona: Fundación CIDOB, págs. 45-78.

Longo, Francisco y Ramio, Carles (2008) La profesionalización del empleo público en América Latina. Barcelona: Fundación CIDOB.

Lopez, Andrea (2005) "Los fundamentos de la Nueva Gestión Pública:lógica privada y poder tecnocrático en el Estado mínimo". En:Thwaites Rey, M. y López,A. (ed.) Entre tecnócratas globalizados y políticos clientelistas: derrotero del ajuste neoliberal en el Estado argentino. Buenos Aires: Prometeo Libros, págs. 71-90.

Mouriño, Miguel (2009) "Modernización y empleo público en la provincia de Buenos Aires". En Aportes para el Estado y la Administración Gubernamental 26: 77-90.

Oszlak, Oscar (2009) "La profesionalización del servicio civil en América Latina: impactos sobre el proceso de democratización" Proyecto OEA-PNUD, La Democracia de ciudadanía: una agenda para la construcción de ciudadanía en América Latina. Descargado de unpan1. un.org/intradoc/groups/public/documents/UB-DPADMUNPAN040085.pdf

Oszlak, Oscar (1999) "Quemar las naves: (o cómo lograr reformas estatales irreversibles)". Revista Aportes para el Estado y la Administración Gubernamental 14.

Orlansky, Dora (2006) Política y burocracia: La reforma del Estado en la Argentina (Tesis Doctoral). Facultad de Ciencias Económicas, Universidad de Buenos Aires.

Pagani, María Laura; Payo, Mariel y Galinelli, Bernardo (2015) Estudios sobre Gestión Pública: aportes para la mejora de las organizaciones estatales en el ámbito provincial (en línea). Disponible en http://www.modernizacion.gba.gob.ar/expertos/sites/default/files/Libro \%20Estudios\%20sobre\%20Gesti $\%$ C3\%B3n\%20P\%C3\%BAblica\%20-\%20ISBNo\%20online.pd]
Piana, Ricardo Sebastián (2012). La reforma en la Administración Pública em la Provincia de Buenos Aires. Su estudio en los años de gestión 2002-2007 (tesis doctorado). Facultad de Ciencias Jurídicas y Sociales, UNLP.

Poblete, Lorena (2013) "Subcontratados por el Estado. Trabajadores autónomos de la administración pública argentina (2002-2007)”. Revista Trabajo y sociedad (21): 459-474.

Pollitt, Cristopher y Bouckaert, Geert (2004). Public Management Reform. A Comparative Analysis. Gran Bretaña: Oxford University Press.

Quintans, Noelia y García, María Elina (septiembre, 2013) "Procesos de selección de personal como política de empleo público: la experiencia de la provincia de Buenos Aires". II Jornadas de RRHH en Instituciones Públicas, Municipalidad de Río Cuarto, Provincia de Córdoba, Argentina.

Randazzo, Florencio (2004) Modernizar el Estado para fortalecer la democracia. Buenos Aires: Editorial Prometeo.

Subsecretaría de Gestión Pública Provincia de Buenos Aires (2005) Plan Trienal de Gestión Pública (2004-2007). Eje 2 Modernización e innovaciones en el ámbito público. Documento $n^{\circ} 2$ (en línea). Disponible en: www.claudiabernazza.com.ar $/ \mathrm{htm} /$ memoria_ssgp/... Dlan_trienal/plan_documentos.htm

Subsecretaría de Gestión Pública Provincia de Buenos Aires (2006) Una Carrera para mejorar el Empleo Público: Documento $n^{\circ} 6.3$ (en línea). Disponible en: www.claudiabernazza.com.ar/htm/memoria_ssgp/Contenido/.../documento6_3.do

Subsecretaría de Gestión Pública Provincia de Buenos Aires (2005) Diagnóstico sobre la relación de empleo público en la Provincia de Buenos Aires. Documento $n^{\circ} 6.1$ (en línea). Disponible en: http://www.claudiabernazza.com.ar/htm/memoria_ssgp/Contenido/Documentos/acciones1_empleo.htm

Villarroel, Amalia Echagüe, Oscar; Volonté, Juan Pablo (Noviembre, 2012) "Las relaciones laborales en el sector público de la Provincia de Buenos Aires". II Congreso Nacional de Relaciones Laborales ACILTRHA / I Congreso Internacional de Integración Laboral Regional de América Latina UITEC. Buenos Aires: ACILTRHA.

\section{Fuentes consultada}

Dirección Provincial de Presupuesto, Provincia de Buenos Aires. http://www. ec.gba.gov.ar/areas/hacienda/Presupuesto/ 
Dirección Nacional de Coordinación Fiscal con las Provincias, Ministerio de Hacienda y Finanzas Públicas de la nación. http://www2.mecon.gov.ar/hacienda/ dncfp/provincial.htm

INDEC Encuesta Permanente de Hogares. http://www.indec.gov.an

\section{Apéndice}

Tabla 1. Regímenes laborales de la Administración Pública de la Provincia de Buenos Aires

\begin{tabular}{|c|c|c|}
\hline Regímenes laborales $(\star)$ & Normativa & $\begin{array}{l}\text { Porcentaje sobre } \\
\text { la dotación total }\end{array}$ \\
\hline 1) Estatuto del Docente & Ley 10.579 & $55,3 \%$ \\
\hline $\begin{array}{l}\text { 2) Estatuto General para los agentes públicos de la } \\
\text { Administración Pública de la Pcia. De Buenos Aires }\end{array}$ & $\begin{array}{l}\text { Ley } 10.430 \text { y sus Decretos } \\
\text { reglamentarios }\end{array}$ & $24,6 \%$ \\
\hline 3) Personal de la Policía de Buenos Aires. & Ley 13.201 y Decreto $1766 / 05$ & $11,8 \%$ \\
\hline 4) Carrera Profesional Hospitalaria & Ley 10.471 & $2,7 \%$ \\
\hline 5) Personal del Servicio Penitenciario & Decreto-Ley 9578/1980 & $2,6 \%$ \\
\hline 6) Personal técnico- gráfico & Ley 10.449 & $3 \%$ \\
\hline 7) Investigador científico-tecnológico & Decreto- Ley 9688/1981. & \\
\hline 8) Personal de Investigación y Desarrollo & Ley 13.487 & \\
\hline 9) Personal del OCABA, Autoridad del Agua y SPAR & Ley 10.384 & \\
\hline 10) Actividades artisticas, técnicas y complementarias & Ley 12.268. & \\
\hline 11) Personal de la Dirección de Vialidad & Ley 10.328 & \\
\hline 12) Clero Oficial & Decreto Ley $8815 / 1977$ & \\
\hline 13) Personal Docente de Minoridad & Ley 10.648 & \\
\hline $\begin{array}{l}\text { 14) Personal Transferido de la Administración General de } \\
\text { Puertos Sociedad del Estado a la Dirección Provincial de } \\
\text { Actividades portuarias }\end{array}$ & ССТ 164/1975, 17/1975, 24/1975. & \\
\hline 15) Personal Ferroviario Transferido & CCT 21/1975, 26/1975Y 27/1975. & \\
\hline $\begin{array}{l}\text { 16) Personal Transferido de AFNE SA por Astilleros Río } \\
\text { Santiago a la Provincia de Buenos Aires }\end{array}$ & ССT $91 / 75$ & \\
\hline 17) Personal transferido de Casinos & $\begin{array}{l}\text { Ley } 22.140 \text { (Estatuto del Empleado } \\
\text { Público nacional, derogado en dicho } \\
\text { ámbito). }\end{array}$ & \\
\hline 18) Personal de OCEBA & CCT 36/1975 & \\
\hline 19) Personal EX EPRE (actual Dirección de Energía) & CCT No $36 / 75$ & \\
\hline
\end{tabular}

Fuente: elaboración propia sobre datos de la Subsecretaria de Gestión Pública (2005)

(*) Los datos son relativos a los empleados del poder ejecutivo, de modo que resultan excluidos el personal de poder legislativo, judicial y bancos.
Tabla 2. Evolución del empleo público de la provincia de Buenos Aires y relación con la población total y la Población Económicamente Activa (PEA) (1990-2015).

\begin{tabular}{|c|c|c|c|c|c|}
\hline Año & $\begin{array}{c}\text { Dotación empleo } \\
\text { público provincial }\end{array}$ & $\begin{array}{c}\text { Variacín porcentual } \\
\text { interanual del empleo } \\
\text { público provincial }\end{array}$ & $\begin{array}{c}\text { Población total } \\
\text { PBA (*) }\end{array}$ & $\begin{array}{c}\text { Porcentaje } \\
\text { Empleados } \\
\text { públicos sobre la } \\
\text { población total } \\
\text { PBA }\end{array}$ & $\begin{array}{c}\text { Empleo } \\
\text { públic/PEA } \\
(\star \star)\end{array}$ \\
\hline 1990 & 287.468 & 1,77 & 12.571 .714 & 2,29 & 6,00 \\
\hline 1991 & 282.680 & $-1,67$ & 12.736 .080 & 2,22 & 5,73 \\
\hline 1992 & 286.255 & 1,26 & 12.897 .142 & 2,22 & 5,61 \\
\hline 1993 & 287.855 & 0,56 & 13.056 .791 & 2,20 & 5,42 \\
\hline 1994 & 287.855 & 0 & 13.216 .914 & 2,18 & 5,33 \\
\hline 1995 & 319.667 & 11,05 & 13.379 .401 & 2,39 & 5,62 \\
\hline 1996 & 323.936 & 1,34 & 13.544 .764 & 2,39 & 5,67 \\
\hline 1997 & 348.788 & 7,67 & 13.634 .681 & 2,56 & 5,87 \\
\hline 1998 & 365.939 & 4,92 & 13.879 .577 & 2,64 & 6,07 \\
\hline 1999 & 404.655 & 10,58 & 14.047 .486 & 2,88 & 6,82 \\
\hline 2000 & 428.408 & 5,87 & 14.047 .486 & 3,05 & 7,06 \\
\hline 2001 & 412.984 & $-3,6$ & 13.755 .993 & 3,00 & 6,91 \\
\hline 2002 & 406.182 & $-1,65$ & 14.290 .241 & 2,84 & 6,71 \\
\hline 2003 & 426.880 & 5,1 & 14.410 .581 & 2,96 & 6,27 \\
\hline 2004 & 438.649 & 2,76 & 14.530 .996 & 3,02 & 6,28 \\
\hline 2005 & 468.474 & 6,8 & 14.654 .379 & 3,20 & 6,69 \\
\hline 2006 & 492.000 & 5,02 & 14.784 .007 & 3,33 & 7,11 \\
\hline 2007 & 521.807 & 6,06 & 14.917 .940 & 3,50 & 7,67 \\
\hline 2008 & 567.405 & 8,74 & $15.052 .177,00$ & 3,77 & 8,24 \\
\hline 2009 & 583.073 & 2,76 & $15.414 .295,90$ & 3,78 & 8,31 \\
\hline 2010 & 618.815 & 6,13 & 15.625 .084 & 3,96 & 8,71 \\
\hline 2011 & 638.440 & 3,17 & 15.838 .755 & 4,03 & 8,96 \\
\hline 2012 & 654.435 & 2,51 & 16.100 .618 & 4,06 & 9,04 \\
\hline 2013 & 651.272 & $-0,48$ & 16.289 .599 & 4,00 & 8,89 \\
\hline 2014 & 646.214 & $-0,78$ & 16.476 .149 & 3,92 & 8,92 \\
\hline 2015 & 677.362 & 4,82 & 16.659 .931 & 4,07 & 9,24 \\
\hline & & & & & \\
\hline & & & & & \\
\hline
\end{tabular}

Fuente: Elaboración propia en base a los datos de la DNCFP y la EPH.

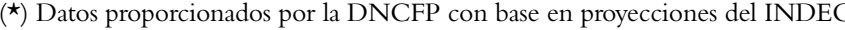

$(\star \star)$ La información relativa a la PEA fue elaborada a partir de la EPH, tomando los datos de seis aglomerados urbanos pertenecientes a la provincia de Buenos Aires durante los diversos trimestres (Partidos del Gran Buenos

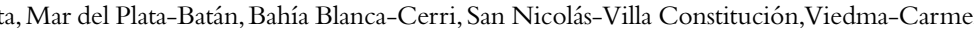


Gráfico 1. Evolución del empleo público provincial (1990-2015)

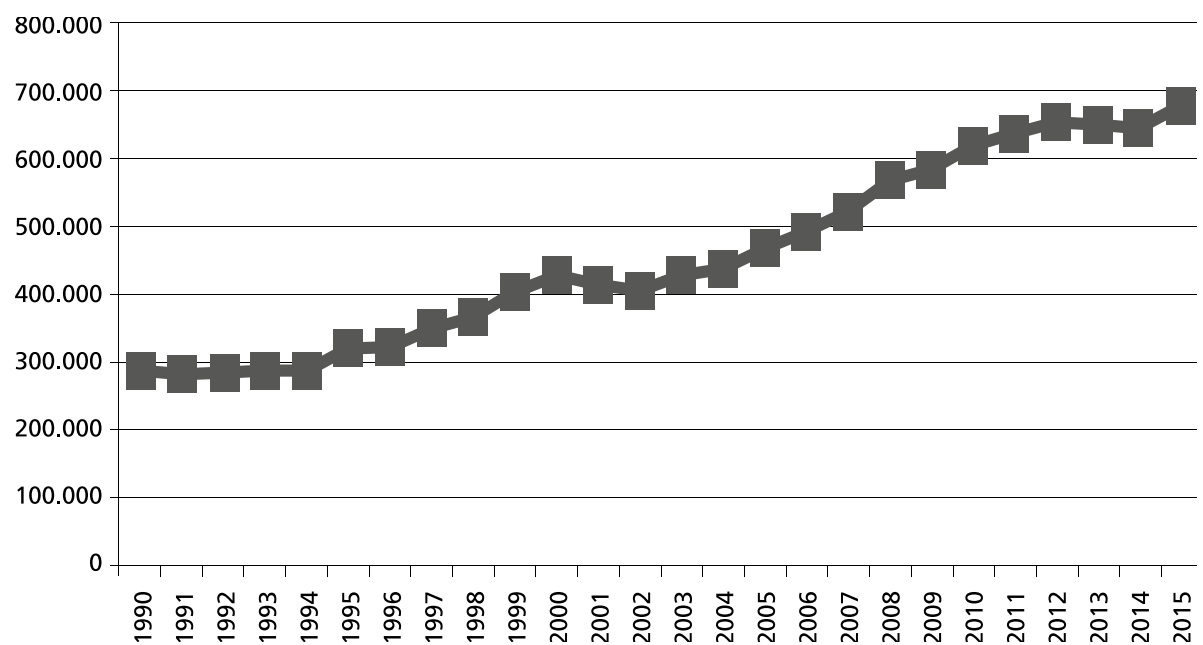

Gráfico 2. Variación porcentual interanual del empleo público provincial (1990-2015)

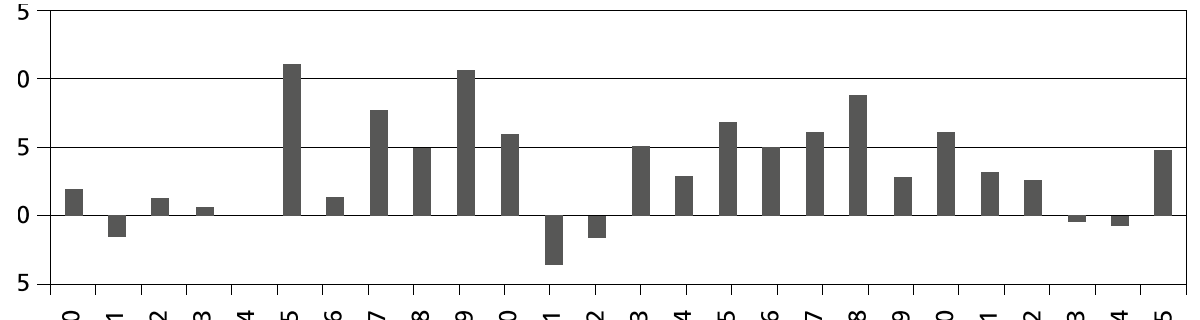

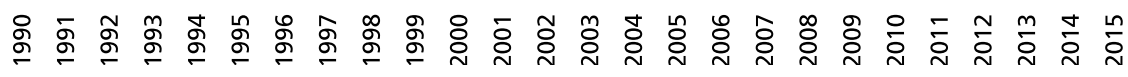

Cómo citar este artículo

Gil García, Magdalena (2017) "Políticas de empleo público en la provincia de Buenos Aires (1991-2016)". Revista Perspectivas de Políticas Públicas vol 7 № 13:

\section{El Instrumento Militar Argentino: reflexiones en torno a su despliegue}

Argentina's Military Instrument: Reflections on its deployment

Sol Gastaldi

UBA/universidad Nacional de la Defensa (UNDEF)

Sergio G. Eissa

UBA/Universidad Nacional de la

Defensa (UNDEF
Fecha de recepción:

15.6.17

Fecha de aceptación:

10.8.17

\section{Resumen}

El despliegue del instrumento militar argentino presenta rasgos que no se condicen con la actitud estratégica adoptada con la sanción de la Ley de Defensa Nacional. Si bien en el año 2005 el gobierno adoptó medidas que dieron inicio a un ciclo de planeamiento estratégico destinado a modernizar el instrumento militar en función de la realidad nacional, regional e internacional, el despliegue permanece como un asunto pendiente que exige su revisión, a fin de conciliar fuerzas armadas capaces de cumplir con éxito su misión principal.

Palabras clave: defensa nacional - instrumento militar argentino - despliegue militar - planeamiento estratégico - defensa no provocativa

\section{Abstract}

The deployment of Argentina's military instrument displays a certain disjunction with regard to the strategic stance adopted in the National Defense Act. Notwithstanding the actions ennacted in 2005 by the National Government, which initiated a cycle of strategic planning addressed at modernizing the military instrument in order to keep it apace with bot national, regional and international realities, its territorial deployment is a pending issue awaiting for revision in order to further the Armed Forces capabilities to succesfully accomplish with their main mission. 